\title{
High gradient limits due to single bunch beam breakup in a collinear dielectric wakefield accelerator
}

\author{
C. Li, ${ }^{1,3}$ W. Gai, ${ }^{1}$ C. Jing, ${ }^{1,4}$ J. G. Power, ${ }^{1}$ C. X. Tang, ${ }^{3}$ and A. Zholents ${ }^{2}$ \\ ${ }^{1}$ High Energy Physics Division, Argonne National Laboratory, Argonne, Illinois 60439, USA \\ ${ }^{2}$ Advanced Photon Source, Argonne National Laboratory, Argonne, Illinois 60439, USA \\ ${ }^{3}$ Department of Engineering Physics, Tsinghua University, Beijing 100084, China \\ ${ }^{4}$ Euclid Techlabs LLC, Solon, Ohio 44139, USA \\ (Received 4 April 2014; published 16 September 2014)
}

\begin{abstract}
A study into the maximum acceleration gradient, $E_{z}^{\max }$, in a collinear dielectric wakefield accelerator due to single bunch beam breakup (SBBU) is presented. The longitudinal wakefield is proportional to charge over radius squared $\left(E_{z} \sim Q / a^{2}\right)$ which implies small $a$ is favorable for fixed $Q$. However, when the transverse wakefield $\left(E_{\perp} \sim Q / a^{3}\right)$ is also considered then the SBBU instability severely limits the charge that can pass through a long structure as required for a large transfer of energy from the drive bunch to the witness bunch and hence limits the sustained acceleration that can be obtained.
\end{abstract}

DOI: 10.1103/PhysRevSTAB.17.091302

PACS numbers: 29.27.Bd, 41.60.-m, 41.75.Lx

\section{INTRODUCTION}

Future large-scale accelerator facilities such as X-ray FEL light sources and linear colliders will significantly benefit from accelerating techniques with high acceleration gradients. In the collinear dielectric wakefield acceleration (DWA) method considered here, a leading, high-charge, $Q$, drive bunch loses energy while traversing a dielectric-lined waveguide and generates wakefields. The longitudinal wakefield, $E_{z}$, is used to accelerate a low-charge witness bunch which trails collinearly through the same structure. While high accelerating gradients in excess of $1 \mathrm{GV} / \mathrm{m}$ have been demonstrated [1] in DWA structures, sustained acceleration, as required for high final witness bunch energy, has not. The final energy is the product of the gradient, $E_{z}$, and the distance, $L$, that the drive bunch and the witness bunch have propagated in the structure. The overall wall-plug efficiency of the accelerator is proportional to the fraction of energy extracted from the drive bunch, $\eta=1-E_{f} / E_{0}$, where $E_{0}$ is initial kinetic energy, $E_{f}$ a final energy and $\eta$ is proportional to the propagation length of drive bunch. For example, consider an $E_{0}=$ $1 \mathrm{GeV}$ drive bunch that generates a gradient of $1 \mathrm{GV} / \mathrm{m}$ and decelerates at $0.5 \mathrm{GeV} / \mathrm{m}$. If it only propagates $L=$ $0.1 \mathrm{~m}$ then $E_{f}=0.95 \mathrm{GeV}$ thus $\eta=5 \%$, which is unacceptable for a large-scale accelerator facility.

As seen above, to obtain both high final witness bunch energy and high efficiency requires a long propagation distance, $L$. However, large longitudinal wakefields are accompanied by large transverse wakefields and these later

Published by the American Physical Society under the terms of the Creative Commons Attribution 3.0 License. Further distribution of this work must maintain attribution to the author(s) and the published article's title, journal citation, and DOI. wakes drive the single bunch beam breakup (SBBU) [2] instability. This causes the loss of the drive bunch in the DWA structure (i.e., short $L$ ) before its energy can be fully extracted. The longitudinal wakefield scales as $E_{z} \propto Q / a^{2}$ where $Q$ is the drive bunch charge and $a$ is radius of the structure channel. This scaling implies that high gradient can be achieved by either increasing $Q$ or decreasing $a$. The transverse wakefield scales as $E_{\perp} \propto Q / a^{3},(\perp=x, y)$ thus decreasing $a$ to raise the gradient will increase the SBBU even more quickly. Since large longitudinal wakefields are desired, efficient control of SBBU is essential for a practical DWA-based facility. SBBU control is complicated by the fact that the drive bunch energy spread is approaching $100 \%$ towards the end of the structure.

In this paper, we derive a scaling law for the high gradient limit of a DWA due to SBBU. Furthermore, we choose quartz as an example to show that it is not possible to operate at high acceleration gradients, close to the material breakdown threshold [1], unless the accelerator is operated at low efficiency.

\section{SBBU CONTROL BY A FODO CHANNEL}

The wakefields generated by a single ultrarelativistic particle in a DWA structure have already been solved $[3,4]$. In our previous paper [5], simulations showed that SBBU effects could be controlled by means of a tapered focusdrift-defocus-drift (FODO) lattice around the DWA structure of inner and outer radius of $a$ and $b$ (Fig. 1). The field gradient of the quadrupoles $\left(B^{\prime}\right)$ decreases along the beam axis to compensate for the energy loss of the drive bunch. In this paper, we extend this work to determine the maximum acceleration gradient achievable as a function of channel radius, $a$, when $\eta$ is considered. In this paper, only the single DWA geometry is considered. For other DWA 


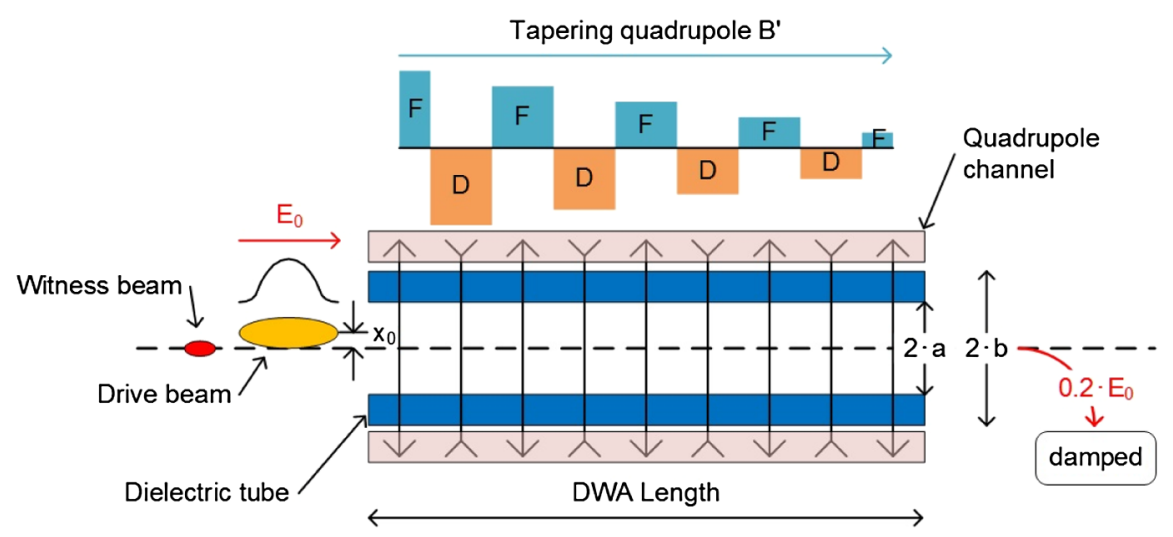

FIG. 1. Schematic of a DWA linac surrounded by a FODO lattice. The quadrupole field gradient $B^{\prime}$ is tapered to match the energy loss of the drive bunch along the linac. The final energy of the drive bunch at the end of the structure is $20 \%$ of its initial energy.

geometries, such as slab geometry, it is difficult to apply strong focusing by quadrupole magnets.

The focusing force of the FODO lattice is periodic and continuous. The length of the quadrupoles in the FODO lattice is constant while the field gradient is tapered to match the drive bunch energy loss due to the longitudinal wakefields,

$$
B^{\prime}(z)=(-1)^{n} B_{0}^{\prime}\left(1-\eta \frac{z}{L}\right)
$$

where $n$ is the quadrupole number along the linac, used to set the sign of the focusing or defocusing force; $B_{0}^{\prime}$ is the quadrupole field gradient; $L$ is the total length of the DWA linac (equal to the length of the FODO lattice); and $\eta$ is called the tapering factor in this context but it is equal to the fraction of energy extracted from above.

\section{A. Two-particle study of SBBU control}

Our method of controlling SBBU in a DWA structure is a generalized version of Balakin-Novokhatski-Smirnov (BNS) damping [6], where a transverse oscillation frequency chirp is imparted from the head to the tail of the bunch to suppress coherent oscillations. We begin by considering a two-particle model of SBBU in the DWA structure imbedded into FODO lattice before presenting the multiparticle simulations results. Assume that both the head, "1", and tail, "2", particles have the same velocity $v_{1}=v_{2} \approx c$ and initial transverse offset $x_{1}(0)=x_{2}(0)=$ $x_{0}$ and angle $x_{1}^{\prime}(0)=x_{2}^{\prime}(0)=0$. The longitudinal wakefields cause both particles to lose energy linearly along $z$, $\gamma_{1}(z)=\gamma_{0}\left(1-\alpha_{1} z\right), \gamma_{2}(z)=\gamma_{0}\left(1-\alpha_{2} z\right)$, where $\alpha_{1}$ and $\alpha_{2}$ are the rates at which the energy decreases. Notice that the different rates of energy loss are a source of difference between BNS damping in a DWA and a conventional linac: the energy chirp across the bunch is growing larger as the bunch propagates along the linac in the former case but it is fixed in the later. The quadrupole field gradient is decreased in proportion to the tail particle's energy for better SBBU control so that $\eta / L=\alpha_{2}$. Finally, we replace discrete focusing with continuous focusing to obtain the equations of motion for the head and tail particles respectively,

$$
\begin{aligned}
& x_{1}^{\prime \prime}-\frac{\alpha_{1}}{1-\alpha_{1} z} x_{1}^{\prime}+k_{\beta}^{2}\left(\frac{1-\alpha_{2} z}{1-\alpha_{1} z}\right) x_{1}=0, \\
& x_{2}^{\prime \prime}-\frac{\alpha_{2}}{1-\alpha_{2} z} x_{2}^{\prime}+k_{\beta}^{2} x_{2}=\frac{e(Q / 2) W_{x}}{\gamma_{0} m c^{2}} \frac{x_{1}}{1-\alpha_{2} z},
\end{aligned}
$$

where $\gamma_{0} m c^{2}$ is the initial particle energy, $k_{\beta}=$ $\sqrt{e B_{0}^{\prime} / 4 \gamma_{0} m c}$ is the initial betatron wave number, $e$ is the elementary charge, $Q$ is the bunch charge, and $W_{x}$ is the transverse wakefield in units $\mathrm{V} /\left(\mathrm{Cm}^{2}\right)$.

Equation (2) does not have exact analytic solutions so that we attempt to give approximate solutions for the trajectories of the head and tail particles,

$$
\begin{aligned}
x_{1}(z) \approx & \frac{x_{0}}{1-\alpha_{1} z} \cos k_{\beta *} z, \\
x_{2}(z) \approx & \frac{x_{0}}{\sqrt{1-\alpha_{2} z}} \cos k_{\beta} z+\frac{e Q W_{x}}{2 \gamma_{0} m c^{2}} \frac{x_{0}}{k_{\beta}^{2}-k_{\beta *}^{2}} \frac{1}{\sqrt{1-\alpha_{2} z}} \\
& \cdot\left(\frac{1}{1-\alpha_{1} z}+\frac{1}{\sqrt{1-\alpha_{2} z}}\right) \sin \left(\frac{k_{\beta}+k_{\beta *}}{2} z\right) \\
& \times \sin \left(\frac{k_{\beta}-k_{\beta *}}{2} z\right),
\end{aligned}
$$

with $k_{\beta *}=k_{\beta}\left[1-3\left(\alpha_{2}-\alpha_{1}\right) z / 4 \pi\left(1-\alpha_{1} z\right)\right]$. Note that when both particles have the same rate of energy decrease $\left(\alpha_{1}=\alpha_{2}\right)$, then Eq. (3) will approach the results presented in Ref. [7] and normal BNS damping can be applied.

Numerical and analytic solutions to the equation of motion [Eq. (2)] are compared in Fig. 2. The numerical solutions are solved by Runge-Kutta methods (ODE45) in MATLAB. The approximate analytic solution [Eq. (3)] is seen to be in reasonable agreement with the numerical solutions [Eq. (2) was solved with the Runge-Kutta method]. In the figure, we used $\gamma_{0}=300, Q=13 \times 10^{-9} \mathrm{C}$, 

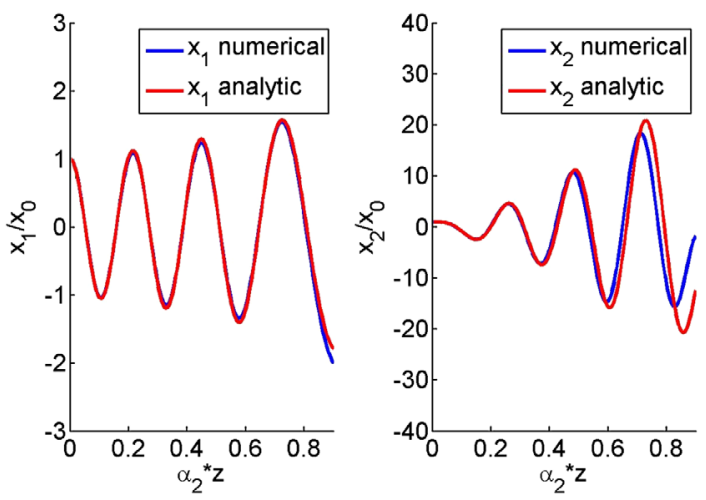

FIG. 2. Numerical and analytic solutions of the equations of motion [Eq. (2)] for both the head (1) and tail (2) particles. When $\alpha_{2} z$ equals 1 , the tail particle has lost all of its energy.

$\alpha_{1}=0.37 \mathrm{~m}^{-1}, \alpha_{2}=0.74 \mathrm{~m}^{-1}, B_{0}^{\prime}=1000 \mathrm{~T} / \mathrm{m}, W_{x}=$ $5 \times 10^{18} \mathrm{~V} /\left(\mathrm{C} \mathrm{m}^{2}\right)$. Note that Eq. (3) is an approximate solution of the particle motion equations (2) and will be invalid when tail particle energy approaches to 0 for large $\alpha_{2} z$ [note $\gamma_{0}\left(1-\alpha_{2} z\right)$ describes the tail particle energy]. Practically, we only want the $80 \%$ of drive bunch energy to be extracted before SBBU occurs as shown in Fig. 1, thus we only need good agreements for $\alpha_{2} z \leq 0.8$.

Inspection of the analytic trajectory of $x_{2}$ (tail) reveals the sources of the amplitude growth. The first term in Eq. (3) for $x_{2}$ corresponds to the betatron oscillation with adiabatic growth, which does not have a strong effect on the amplitude growth until $z$ approaches to $1 / \alpha_{2}$. The second term for $x_{2}$ is due to the wakefield force from the head $\left(x_{1}\right)$ particle and reveals beating with a maximum amplitude growth when $z=\pi / \Delta k_{\beta}$, where $\Delta k_{\beta}=k_{\beta}-k_{\beta *}$. Preliminary work has shown that other distributions, with higher transformer ratios, may propagate longer distances when given an initial energy chirp. (The specific shape plays an important role in the BBU control as well.) This will be presented in future work.

Controlling SBBU in a DWA is now seen as a matter of controlling the amplitude growth of the tail particle due to the wakefield force [second term in Eq. (3)]. If the longitudinal wakefields are not strong $\left(\alpha_{1}\right.$ and $\alpha_{2}$ are small), it will perform a beating pattern. However we want to study the high gradient limits here, so the longitudinal wakefields are strong enough and the tail particle will exhaust its energy with only a small propagation length $L_{\text {prop }}$ before reaching the first beating point. Therefore $\Delta k_{\beta} z$ is much smaller than $\pi / 2$ when $z \leq L_{\text {prop }}$. Making the approximation $\sin \left(k_{\beta}-k_{\beta *}\right) z / 2 \approx\left(k_{\beta}-k_{\beta *}\right) z / 2$, we can express the amplitude of the tail, $x_{2}(z)$, as

$$
\begin{aligned}
A(z)= & \frac{e Q W_{x} x_{0}}{2 \gamma_{0} m c^{2} k_{\beta}} \frac{z}{2} \frac{1}{\sqrt{1-\alpha_{2} z}} \frac{1}{2-\frac{3\left(\alpha_{2}-\alpha_{1}\right) z}{4 \pi\left(1-\alpha_{1} z\right)}} \\
& \times\left(\frac{1}{\sqrt{1-\alpha_{2} z}}+\frac{1}{1-\alpha_{1} z}\right) .
\end{aligned}
$$

We now present a case study of a high efficiency DWA structure with $\eta=80 \%$, thus $\alpha_{2} L=\eta=0.8$. The head particle $(Q / 2)$ sees half of the maximum longitudinal wakefield generated by itself, thus $\alpha_{1} L=0.5 \alpha_{2} L=0.4$. The total propagation length is $L=\eta \gamma_{0} m c^{2} / e E_{z}^{\mathrm{dec}}$; the deceleration gradient is $E_{z}^{\mathrm{dec}}=(Q / 2) W_{z}$. The amplitude of the tail particle $(Q / 2)$ at $z=L$ is

$$
A(L)=1.896 \frac{(Q / 2) W_{x} x_{0}}{E_{z}^{\operatorname{dec}} k_{\beta}}=1.896 \frac{W_{x} x_{0}}{W_{z} k_{\beta}} .
$$

Thus, the amplitude at the end of the DWA linac is a function of the following five parameters: transverse wakefields $W_{\mathrm{x}}$, longitudinal wakefields $W_{z}$, initial energy $\gamma_{0}$, initial offset $x_{0}$, and initial quadrupole field gradient $B_{0}^{\prime}$.

Next, we parametrize Eq. (5) in terms of only the bunch charge $Q$ and the channel radius, $a$, in order to develop a scaling law for the maximum acceleration gradient.

(1) Wakefields.-The ratio of the outer and inner radii of the DWA structure $b / a$ was kept so as to keep the ratio of fundamental to higher-order modes constant. Thus,

$$
W_{x}=W_{x 1} \cdot a_{1}^{3} / a^{3}, W_{z}=W_{z 1} \cdot a_{1}^{2} / a^{2} .
$$

(2) Free betatron oscillation in the FODO channel.First we have the relation between the quadrupole focal length and the FODO cell length with a betatron phase per cell $\varphi=90^{\circ}$ as $L_{0} / 2 f=\sin (\varphi / 2)=\sqrt{2} / 2$ [8], where $2 L_{0}$ is the length of a FODO cell, $f=\gamma_{0} m c / e B_{0}^{\prime} L_{q}$ is the focal length of a quadrupole and $L_{q}$ is quadrupole length. Because stronger focusing in the FODO lattice allows for improved SBBU control, we take the upper limit that $L_{q}=L_{0}$ and $B^{\prime}=B_{\text {sat }} / a$, where $B_{\text {sat }}$ is saturation magnetization at pole tips and is equal to 1.0 Tesla in this paper. (A new type of focusing quadrupole whose $B^{\prime}$ may reach $3000 \mathrm{~T} / \mathrm{m}$ is under development [9].) Then one can write

$$
L_{q}=L_{0}=\sqrt{\frac{\sqrt{2} \gamma_{0} m c a}{e B_{\mathrm{sat}}}}, k_{\beta}=\sqrt{\frac{e B_{\mathrm{sat}}}{4 \gamma_{0} m c a}} .
$$

(3) Initial $x_{0}$ relative to axis.-We have assumed that the $x_{0}$ is proportional to a bunch rms size $\sigma_{x}$, i.e., $\sim 0.5 \sigma_{x}$. We assume the dependence of emittance on bunch charge is approximately linear, $\varepsilon_{x n}=C_{0} Q$, with a typical value of the coefficient $C_{0}=\varepsilon_{x n} / Q=1 \mu \mathrm{m} / \mathrm{nC}=1000 \mathrm{~m} / \mathrm{C}$. Thus,

$$
\left.\begin{array}{rl}
x_{0} & =0.5 \sigma_{x}=0.5 \sqrt{\varepsilon_{x n} \beta_{x} / \gamma_{0}} \\
\varepsilon_{x n} & =C_{0} Q \\
\beta_{x} & =\lambda_{\beta} / 2 \pi=2 L_{0} / \varphi=4 L_{0} / \pi
\end{array}\right\}
$$


Applying the above equations to Eq. (5) we have an expression that relates the amplitude of the tail $\left(x_{2}\right)$ at $z=L$ to the charge and the channel radius,

$$
A(Q, a)=\frac{4.135}{\sqrt{\pi}} \frac{a_{1} W_{x 1}}{W_{z 1}}\left(\frac{\gamma_{0} m^{3} c^{3} C_{0}^{2}}{e^{3} B_{\mathrm{sat}}^{3}}\right)^{1 / 4} \frac{Q^{1 / 2}}{a^{1 / 4}} .
$$

Propagation requires that $A(Q, a) \leq a$, i.e., that the bunch does not hit the wall. Finally, one can determine an analytic expression for the maximum charge that can be propagated through the DWA structure,

$$
Q_{\max }(a)=\frac{\pi}{17.10}\left(\frac{W_{z 1}}{a_{1} W_{x 1}}\right)^{2}\left(\frac{e^{3} B_{\mathrm{sat}}^{3}}{\gamma_{0} m^{3} c^{3} C_{0}^{2}}\right)^{1 / 2} a^{5 / 2} .
$$

All the parameters in the above equation are in SI units.

\section{B. Structure parameters}

Before we can numerically determine how $Q_{\max }$ scales with $a$, several steps remain. First, we numerically calculate the wakefields, $W_{z}$ and $W_{x}[3,4]$ for a particular value of $a$; we call this the reference case. For the reference case, we choose a DWA structure with $a_{1}=1.0 \mathrm{~mm}, b_{1}=1.06 \mathrm{~mm}$ and $\varepsilon=3.75$ (as an example, we use quartz as the dielectric material) which uniquely determines the fundamental frequency, $f_{1}=300 G \mathrm{~Hz}$. Numerical calculation gives a maximum transverse wake inside the Gaussian bunch as $W_{x 1}=4.5 \mathrm{MV} /(\mathrm{nC} \mathrm{m} \mathrm{mm})$ and a maximum deceleration wake $W_{z 1}=8.0 \mathrm{MV} /(\mathrm{nCm})$ shown in Fig. 3 .

Next we explain how the ratio $b / a$ and dielectric constant $\varepsilon$ affects wakefields and BBU. By numerical study we found that thinner dielectric layer (which leads to higher group velocity $v_{\mathrm{g}}$ ) would be better for $\mathrm{BBU}$ control because transverse wakefields will be weaker at higher group velocity case [Fig. 4(a)]. Thus we choose these particular values as $b / a=1.06$ and $v_{\mathrm{g}}=0.82 c$. We

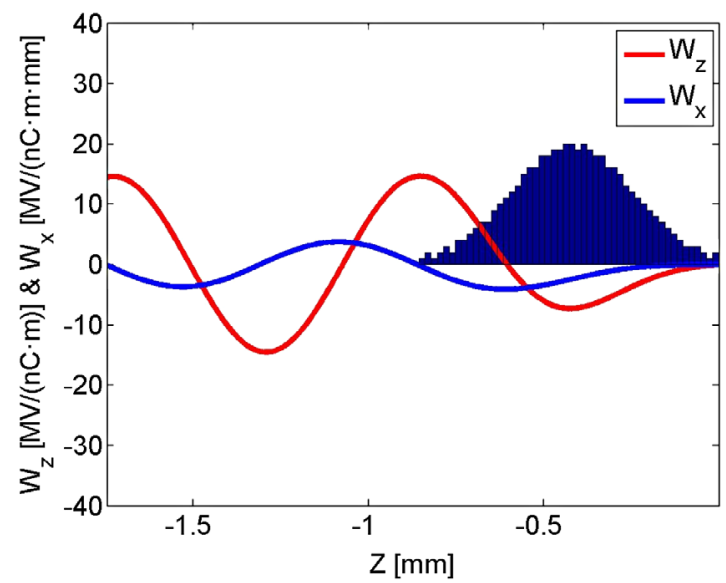

FIG. 3. Longitudinal and transverse wakefields generated by a Gaussian bunch in a DWA structure with $a_{1}=1.0 \mathrm{~mm}, b_{1}=$ $1.06 \mathrm{~mm}$ and $\varepsilon=3.75$. The bunch moves towards the right.
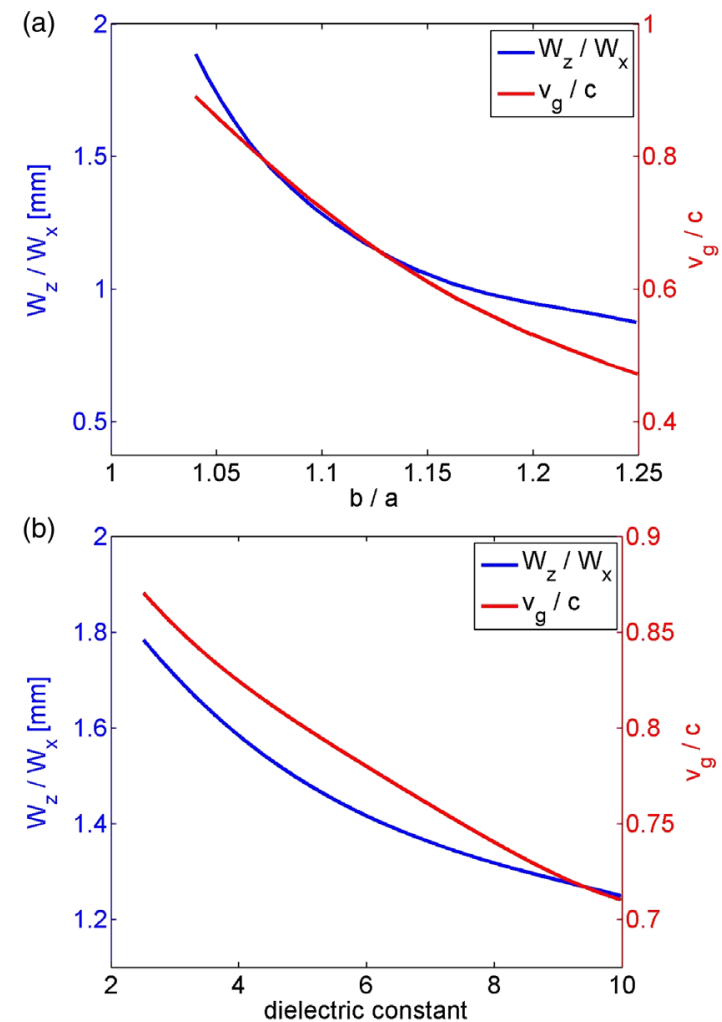

FIG. 4. Ratio of longitudinal and transverse wakefields $W_{\mathrm{z}} / W_{\mathrm{x}}$ (blue line) and group velocity (red line) as functions of $b / a$ (up) and dielectric constant (down). Both $b / a$ and dielectric constant can affect group velocity. Higher group velocity leads to weaker transverse wakefields and weaker BBU effects.

are afraid that an even thinner dielectric layer may be a big challenge for manufacturing. The dielectric constant can also affect the group velocity and BBU effects. Lower dielectric constant $\varepsilon$ helps to reduce transverse wakefields and BBU effects [Fig. 4(b)].

These numerical values of the reference case are used to scale the wakefields according to Eq. (6). Next, we set the rms length of a Gaussian bunch according to $\sigma_{z} / \lambda=0.2$ for all cases and for the reference case we have $\lambda_{1}=c / f_{1}=1 \mathrm{~mm}$. This value of $\sigma_{z} / \lambda$ achieves high acceleration gradient and high transformer ratio [10]. The initial bunch energy is taken as a constant, $150 \mathrm{MeV}$, so $E_{0}$ is not scaled with $a$. Given the above values, one can use Eq. (10) to find the maximum charge is $17 \mathrm{nC}$. Finally, we substitute the above values into Eq. (10), to obtain the two-particle model prediction of the scaling of charge with radius,

$$
Q_{\max }[\mathrm{nC}] \approx 17.1 \times a[\mathrm{~mm}]^{5 / 2} .
$$

\section{Simulations results}

Multiparticle simulations were done with a user-written particle pushing code to simulate beam dynamics including SBBU in the DWA structure surrounded by a FODO lattice. 


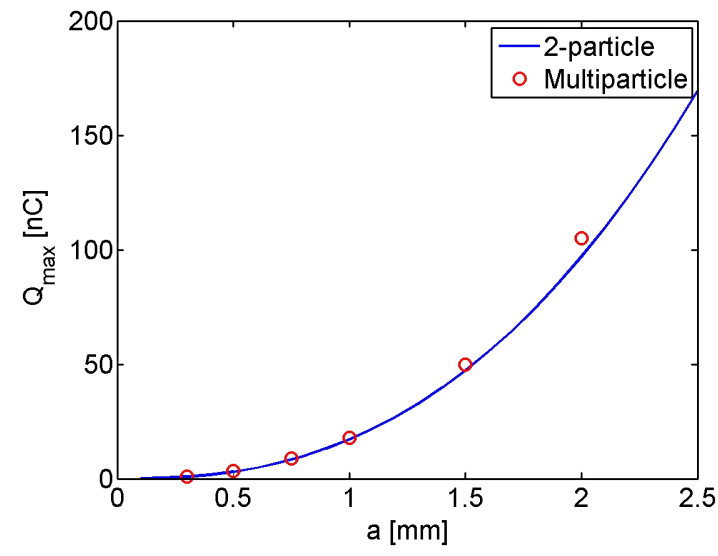

FIG. 5. Maximum bunch charge as a function of the radius in the DWA linac for $\eta=80 \%$. The blue line is the two-particle model result and the red circles are the multiparticle beam dynamics simulation results.

The basic mathematical model is the same as Ref. [5]. It applies the force due to wakefield and quadrupoles to the macroparticles and tracks them in the two-dimensional coordinates $(x, z)$. The wakefields excited by a single particle in the DWA were decomposed into discrete but infinite numbers of waveguide modes ( $\mathrm{mn}$ in Ref. [3,4]). Unless specifically mentioned, the wakefield calculations used only the first three modes of both the monopole and dipole wakes (due to the finite length of the bunch) but convergence studies were done in all cases. The space charge effects are much weaker than wakefield forces and are excluded in our simulations. Based on simple estimations, the transverse space charge force is at least smaller than transverse wakefields force by one power of 10 . We have also done some simulations in ASTRA. The results show good agreements with and without space charge. The only difference is that magnet focusing forces should be a little bit stronger to compensate space charge forces. Our simulations were also checked to be in good agreement with both Elegant [11] and BBU3000 [12].

The scaling of charge with radius ( $Q_{\max }$ vs $a$ ) according to multiparticle simulations was determined for several $\operatorname{radii}(a[\mathrm{~mm}]=0.3,0.5,0.75,1.0,1.5$, and 2.0). Simulation results (Fig. 5, red circles) are in good agreement with Eq. (11) from the two-particle model (Fig. 6, blue line).

\section{High gradient limits}

The scaling of the maximum acceleration gradient, $E_{z}^{\max }$, with structure radius, $a$, for both the two-particle model and the numerical simulations can now be simply obtained from their above charge scaling results. Given $E_{z}^{\max }=Q_{\max } W_{z}^{\text {acc }}$, then for the reference case $\left(a_{1}=1.0 \mathrm{~mm}, f_{1}=300 \mathrm{GHz}\right.$, $Q_{\max }=17 \mathrm{nC}$ and $\left.W_{z}^{\text {acc }}=2 W_{z 1}=17 \mathrm{MV} /(\mathrm{nCm})\right)$ we find $E_{z}^{\max }=290 \mathrm{MV} / \mathrm{m}$. This result can be used with $W_{z} \sim$ $Q / a^{2}$ to obtain the two-particle model prediction of the scaling of maximum gradient with radius,

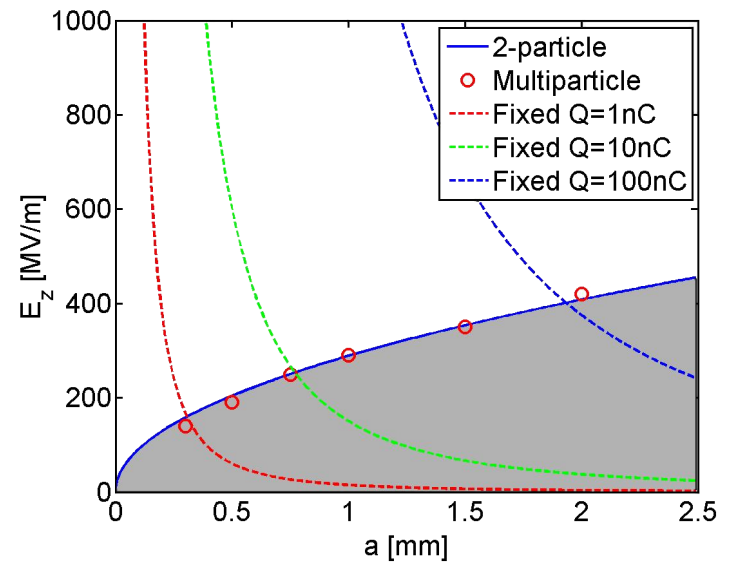

FIG. 6. Maximum acceleration gradient as a function of the radius in the DWA linac for $\eta=80 \%$. The blue line is the maximum gradient and the grey area underneath is the region of permissible gradients. [The dashed lines show the gradient scaling $\left(Q / a^{2}\right)$ when SBBU is ignored.]

$$
E_{z}^{\max }[\mathrm{MV} / \mathrm{m}] \approx 290 \times \sqrt{a[m m]} .
$$

Comparison of the scaling of $E_{z}^{\max }$ with radius shows good agreement between the simulation results (Fig. 6, red circles) and the two-particle model (Fig. 6, blue line). For reference, we plot the gradient scaling with $a$ when SBBU is ignored for various fixed charges using, $W_{z} \sim Q / a^{2}$ (dashed colored lines in Fig. 6).

The above results [Fig. 6 and Eq. (12)] are the key results of this paper and can be used to guide the accelerator designer in the choice of the DWA structure. If high charge is available (e.g., Ref. [13] has drive bunch of $Q=100 \mathrm{nC}$ ) then a maximum gradient of $400 \mathrm{MV} / \mathrm{m}$ with structure radius $a=2 \mathrm{~mm}$ is obtainable. At the other end of the spectrum, if the drive bunch charge available is only $1.0 \mathrm{nC}$, as is typical for an SRF linac, then the maximum gradient is $150 \mathrm{MV} / \mathrm{m}$ for $a=0.3 \mathrm{~mm}$.

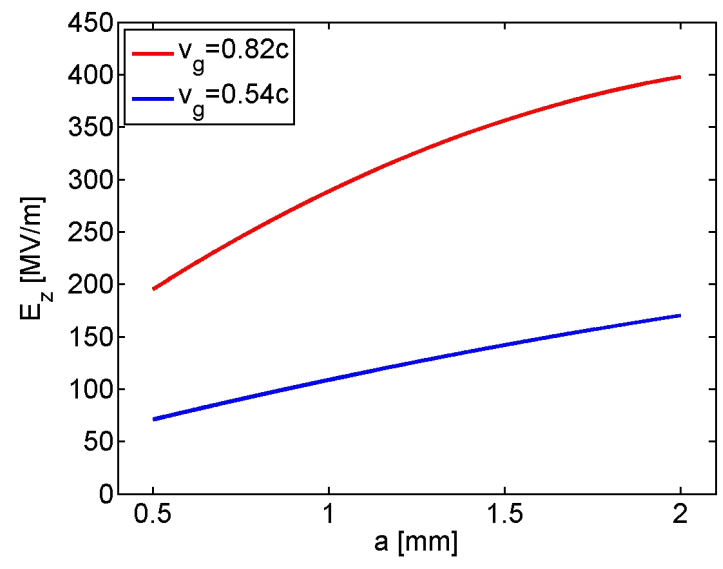

FIG. 7. Maximum acceleration gradient as a function of the radius in the DWA linac for a different group velocity case. $v_{\mathrm{g}}=$ $0.82 c$ and $v_{\mathrm{g}}=0.54 c$ cases are corresponding to $b / a=1.06$ and $b / a=1.2$, respectively. 


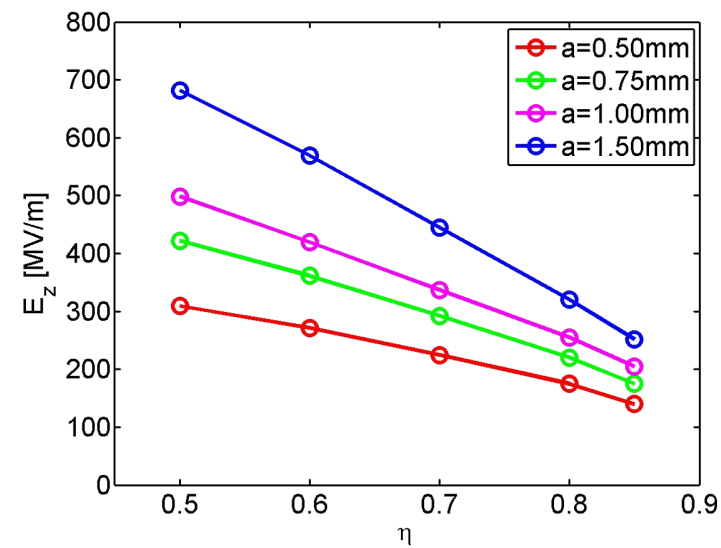

FIG. 8. Maximum acceleration gradient as a function of efficiency $\eta$. Higher gradient limits can be achieved by sacrificing the efficiency.

For other structure radii ratio $b / a$ and dielectric material, our simulation results show that smaller $b / a$ and lower dielectric constant $\varepsilon$ can lead to higher group velocity and higher $W_{z} / W_{x}$, which help to control SBBU effects and achieve higher gradient limits [Fig. 7].

The maximum acceleration gradient, $E_{z}^{\max }$, can be increased if one is willing to sacrifice the efficiency (by lowering the requirement for $\eta$ ) and the total energy gain of the accelerator. This is because the charge must be raised to increase the gradient $E_{z 0}=Q W_{z}$ which increases SBBU and therefore reduces the propagation length, $L$. For example, $E_{z}^{\max }$ can be increased from $\sim 290$ to $\sim 450 \mathrm{MV} / \mathrm{m}$ (Fig. 8) by doubling the charge in the reference case ( $a=1.0 \mathrm{~mm}$ ). However, $L$ is then reduced from 85 to 35 $\mathrm{cm}$, thus $\eta$ drops from $80 \%$ to $60 \%$ and the total energy gain decreased from 245 to $157 \mathrm{MeV}$.

\section{MAGNET ALIGNMENT TOLERENCE}

BBU control presented in the paper is based on an ideal quad channel. In practice, many factors need to be considered. For example, because of the large quadrupole strength, its alignment becomes critical. Following the method in [14] (without consideration of transverse wakefields), let us consider a random sequence of magnet misalignment $\delta x_{i}$; the ensemble average displacement due to the misalignment is

$$
\left\langle x^{2}(s)\right\rangle=\sum_{i}\left\langle(\delta x)_{i}^{2}\right\rangle \frac{\beta_{x}\left(s_{i}\right) \beta_{x}(s)}{f_{x}^{2}\left(s_{i}\right)} \frac{\gamma\left(s_{i}\right)}{\gamma(s)} \sin ^{2} \varphi\left(s, s_{i}\right),
$$

where $s_{i}$ and $s$ are locations of each quadrupole and observation, $f_{x}, \beta_{x}$ and $\gamma$ are the focal length, beta function and Lorentz factor at each quadrupole magnet, and $\varphi\left(s, s_{i}\right)$ is the phase advance between $s_{i}$ and $s$. Because $B^{\prime}$ of the FODO channel is tapered to compensate the energy decreasing, $f_{x}$ and $\beta_{x}$ are constant in the DWA linac, denoted as $f_{x 0}$ and $\beta_{x 0}$. Consider a uniform rate of energy decrease, $\gamma(s)=\gamma_{0}(1-\eta s / L)$, and a large number, $N$, of magnets, then we can replace the sum by an integral to obtain the rms displacement at the end of the lattice $(s=L)$,

$$
x(L)_{r m s}=\frac{\beta_{x 0}}{f_{x 0}} \delta_{x, r m s} \sqrt{\frac{(2-\eta) N}{4(1-\eta)}},
$$

where $\eta$ is the fraction of energy extracted from the drive bunch, and $\delta_{x, r m s}$ is rms alignment error. Because we want the displacement at the end of the lattice to be smaller than the beam size there $x(L)_{r m s} \leq \sigma_{x}(L)$ and the beam envelope should be smaller than the channel radius $3 \sigma_{x}(L) \leq a$, the tolerance of random magnet misalignments is given by

$$
\delta_{x, r m s} \leq \frac{f_{x 0}}{\beta_{x 0}} \frac{a}{3} \sqrt{\frac{4(1-\eta)}{(2-\eta) N}} .
$$

For our reference case study (beam aperture $2 a=2 \mathrm{~mm}$ ), the above parameters are given as $f_{x 0}=1.4 \mathrm{~cm}, \beta_{x 0} \approx$ $0.1 \mathrm{~m}, \eta=80 \%$ and $N \approx 20$, then the tolerance is estimated as $8.5 \mu \mathrm{m}$. The tolerance requirement is even tighter when transverse wakefields are considered. For the same case, the numerical simulation shows that a $5 \mu \mathrm{m}$ randomly misalignment will lead to a $30 \%$ reduction of the propagation length. The tolerance can be remediated by a slight decrease of the operational gradient.

\section{SUMMARY}

In summary, collinear wakefield acceleration in the dielectric waveguide has the potential to enable future accelerator science but the SBBU instability imposes a significant limit on the highest acceleration gradient and must be carefully controlled with a focusing channel. Since the achievable focusing is limited by the attainable magnetic field at the tip of the magnetic pole, our study shows that the maximum accelerating gradient increases with structure radius $\sim \sqrt{a}$. This trend is the opposite of what would be expected by using the simple scaling law, $E_{z} \sim Q / a^{2}$.

\section{ACKNOWLEDGMENTS}

This work is supported by the U.S. Department of Energy Office of Science under Contract No. DE-AC0206CH11357, and National Natural Science Funds for Distinguished Young Scholar of China under Contract No. 10925523.

[1] M. C. Thompson et al., Phys. Rev. Lett. 100, 214801 (2008).

[2] T. P. Wangler, Principles of RF Linear Accelerators (John Wiley \& Sons Inc., New York, 1998), p. 339.

[3] M. Rosing and W. Gai, Phys. Rev. D 42, 1829 (1990). 
[4] K. Y. Ng, Phys. Rev. D 42, 1819 (1990).

[5] W. Gai, A. D. Kanareykin, A. L. Kustov, and J. Simpon, Phys. Rev. E 55, 3481 (1997).

[6] V. Balakin, A. Novokhatsky, and V. Smirnov, in Proceedings of the 12th International Conference on High Energy Accelerators (Fermilab, Batavia, IL, 1983), p. 119.

[7] A.W. Chao, Physics of Collective Beam Instability in High Energy Accelerators (Wiley, New York, 1993), p. 139.

[8] H. Wiedemann, Particle Accelerator Physics, 3rd ed. (Springer, New York, 2007), p. 241.

[9] R. Candler, "Microscale Magnetic Flux Sources for Electron Beam Manipulation," in Proceedings of the Workshop on Physics and Applications of High Brightness Beams, San Juan, Puerto Rico, 2013, http://pbpl.physics.ucla.edu/ HBEB2013/Talks/RobCandlerHBEB13.pdf.

[10] J. G. Power, W. Gai, A. Kanareykin, and X. Sun, in Proceedings of the Particle Accelerator Conference, Chicago, IL, 2001 (IEEE, New York, 2001), p. 114.

[11] M. Borland, Report No. APS LS-287, 2000.

[12] http://www.euclidtechlabs.com/product/bbu3k.php.

[13] M. Conde et al., in Proceedings of the 23rd Particle Accelerator Conference, Vancouver, Canada, 2009 (IEEE, Piscataway, NJ, 2009), p. 2923.

[14] R. D. Ruth, Report No. SLAC-PUB-4436, 1987. 